\title{
Corrigendum
}

\section{Anxious-Retarded Depression: Relation with Plasma Vasopressin and Cortisol}

\author{
Remco FP de Winter, Albert M van Hemert, Roel H DeRijk, Koos H Zwinderman, Ank C Frankhuijzen- \\ Sierevogel, Victor M Wiegant and Jaap G Goekoop
}

Neuropsychopharmacology (2003) 28, 610. doi:I0.1038/sj.npp. 1300154

Correction to: Neuropsychopharmacology (2003) 28, 140147. doi:10.1038/sj.npp.1300002

The authors wish to apologize for the errors made in the above paper. The corrected sections of text are produced below:

\section{INTRODUCTION}

...We therefore compared the AVP levels and AVP-cortisol correlations in patients with anxious-retarded-melancholic depression and in the category of all other depressed patients.

\section{DISCUSSION}

In patients with melancholic depression $(65 \%$ of the patients had anxious-retarded depression)...

\section{Plasma Concentrations of Neuroactive Steroids before and after Repetitive Transcranial Magnetic Stimulation in Depression}

Frank Padberg, Flavia di Michele, Peter Zwanzger, Elena Romeo, Giorgio Bernardi, Cornelius Schüle, Thomas C Baghai, Robin Ella, Augusto Pasini and Rainer Rupprecht

Neuropsychopharmacology (2003) 28, 610-6I I. doi:I0.1038/sj.npp. I300I55

Correction to: Neuropsychopharmacology (2002) 27, 874878.

Recently, we published a report on the effects of repetitive transcranial magnetic stimulation (rTMS) on plasma levels of neuroactive steroids in major depression (Padberg et al, 2002). We meanwhile noticed that the progesterone levels reported in Figure 2 in this paper were above those usually found in human plasma. We therefore have reanalyzed our GC/MS data. By doing so we found that, when switching from steroid analysis by GC/MS from the electron impact (EI) mode that was employed in our previous study in depression (Romeo et al, 1998) to the negative chemical ionization (NCI) mode, we made a mistake with the calculation of progesterone concentrations. In our paper (Padberg et al, 2002) we reported on calculations based on 
a
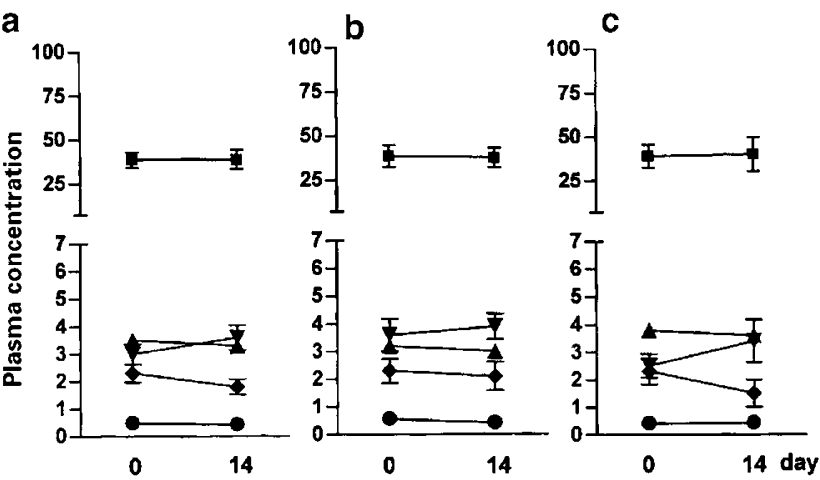

$\rightarrow$ DHEA

$\rightarrow-3 \alpha, 5 \alpha-T H P$

$\pitchfork$ Progesterone

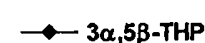

the ions that are characteristic for progesterone out of which the ion $m / z 178$ represents also pregnenolone which partially coelutes with progesterone. This explains why the progesterone values reported were too high. We now recalculated progesterone excluding $m / z$ 178. The corrected mean progesterone concentrations are below $5 \mathrm{nmol} / \mathrm{l}$ as usually found in the literature. The other steroid concentrations reported (Padberg et al, 2002) are correct. We seriously apologize for this error. Nevertheless, we would like to emphasize that the recalculated progesterone data do not change the conclusions of this study that treatment with rTMS for 2 weeks does not affect plasma concentrations of neuroactive steroids in depressive patients.

\section{REFERENCES}

Padberg F, di Michele F, Zwanzger P, Romeo E, Bernardi G, Schule $C$ et al (2002). Plasma concentrations of neuroactive steroids before and after repetitive transcranial magnetic stimulation (rTMS) in depression. Neuropsychopharmacology 27: 874-878.

Romeo E, Ströhle A, Spalletta G, di Michele F, Hermann B, Holsboer $\mathrm{F}$ et al (1998). Effects of antidepressant treatment on neuroactive steroid concentrations in major depression. Am J Psychiatry 155: 910-913.

\title{
Amisulpride vs Risperidone in Chronic Schizophrenia: Results of a 6-Month, Double-Blind Study
}

\author{
D Sechter, J Peuskens, O Fleurot, W Rein and Y Lecrubier the Amisulpride Study Group
}

Neuropsychopharmacology (2003) 28, 610-6II. doi:10.1038/s..npp. I300 I74

Correction to: Neuropsychopharmacology (2002) 27, 10711081.

The authors wish to apologize for errors made in the above paper. The corrected section of text is produced below.

\section{EFFICACY}

Except where stated, efficacy data are presented using the 'maintenance' population and the last-observation-carried forward (LOCF) procedure (all other analyses yielded similar results).

Amisulpride was demonstrated to be not inferior to risperidone with respect to the primary efficacy parameter.
The PANSS total score decreased markedly in both groups (from $91.1 \pm 13.0$ at baseline to $58.9 \pm 22.6$ after 6 months in the amisulpride group and from $92.5 \pm 12.2$ to $61.1 \pm 20.9$ in the risperidone group) (Figure 1). The noninferiority analysis showed that the decrease in the PANSS total score from baseline was not inferior with amisulpride as compared to risperidone (90\% two-sided confidence interval $[-5.6 ; 4.0])$. The two-sided $95 \%$ confidence interval also confirms the noninferiority of amisulpride compared with risperidone (Table 3). Similar results were observed in the ITT overall population (from $91.7 \pm 12.6$ at baseline to $64.8 \pm 25.0$ after 6 months in the amisulpride group and from $92.9 \pm 12.3$ to $66.8 \pm 24.1$ in the risperidone group, $90 \%$ two-sided confidence interval $[-5.4 ; 3.7])$. 